\title{
Personalidad y diferencias sexuales: El papel del sexo, la edad y la experiencia
}

\author{
D. AVIA, J. M. CARRILlO, N. ROJO \\ Universidad Complutense de Madrid
}

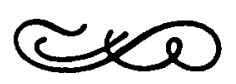

\begin{abstract}
Resumen
Este trabajo es una réplica parcial de la investigación de Snyder y cols. (9186), que relacionó orientaciones ante la sexualidad (actitudes y conductas) con puntuaciones en la escala de $A O$. 265 estudiantes de Psicología respondieron a la encuesta de sexualidad de los autores citados y a la escala de $A O$ de Snyder. El sexo, la edad y la $A O$, en menor medida, predijeron las respuestas al cuestionario, siendo los bombres de más edad y los altos en $A O$ los de una sexualidad menos restringida. Mientras que las diferencias en los sujetos con experiencia de coito se explicaban sobre todo por la variable de personalidad, en los sujetos sin esa experiencia fue el sexo el más determinante. La importancia de la experiencia en el establecimiento de actitudes y conducta sexual en ambos sexos va en contra de la reciente interpretación biológica de la $A O$ de Snyder y su grupo.
\end{abstract}

Palabras clave: Sexualidad, Auto-observación.

\section{Personality and Sexual Differences: the role of sex, age and experience}

\begin{abstract}
This study is a partial replication of Snyder's $E$ cols. research in wich sexual orientation (attitudes and behaviors) was related to scores in the Self-Monitoring scale. 265 Psychology students answered the sexuality survey of the above authors and also filled Snyder's SM scale. Sex, age and to lesser extent Self-Monitoring, predicted answers to the survey, with men, older subjects and bigh self-monitors showing a less restricted orientations towards sexuality. While for subjects with coital experience differences were explained mainly for the personality variable, for subjects without that experience was sex the most determinant variable. The importance of experience in the maintenance of sexual attitudes and behaviors in both sexes contradicts Snyder's and cols. recent interpretation of SM in biological grounds.
\end{abstract}

Keywords: Sexual behavior, Self-Monitoring.

Agradecimientos: Este trabajo es parte de la investigación del mismo nombre financiada por una ayuda del Instituto de la Mujer en Mayo de 1987.

Dirección del autor: D. Avia, Facultad de Psicología, Universidad Complutense, Campus de Somosaguas, Madrid.

(C) 1990 by Aprendizaje, Revista de Psicologia Social, 1990, S (1), 7-22. ISSN: 0213-4748 


\section{INTRODUCCION}

El trabajo que hemos desarrollado se centra en las repercusiones de una variable de personalidad, la Auto-Observación o Self-Monitoring, que se inserta en una larga tradición psicológica, especialmente en el modelo dramatúrgico (Goffman, 1959; Brissett y Edgley, 1975; Snyder, 1987). Los trabajos de Goffman, antecedentes remotos de la investigación que hemos realizado, resaltaron los elementos expresivos de la comunicación humana, tratados según los principios del arte dramático, partiendo de que una motivación básica en los actores sociales es la tendencia a controlar la conducta humana mediante la autoexpresión. La investigación reciente en psicología de la Personalidad ha estudiado las diferencias interpersonales en el grado en que las personas pueden mantener un control sobre su conducta expresiva, las representaciones adecuadas a distintos contextos y los propios signos emocionales no verbales. El constructo psicosocial que engloba esas diferencias es la variable auto-observación definida en 1974 por M. Snyder. Los sujetos altos en $\mathrm{AO}$ se caracterizaron como aquellos individuos que sufren una mayor impacto de las situaciones sociales, presentan más especificidad situacional, hasta el punto de funcionar como distintas personas en distintas situaciones, y menor correspondencia entre sus actitudes y conductas. Estos individuos pueden mostrar alta coherencia expresiva: puesto que la representación social a menudo exige un patrón prototípico que encaja y es adecuado para diversas situaciones, (el del individuo extravertido y controlado), en general, el individuo alto en $\mathrm{AO}$ se comportará con arreglo a ese prototipo. Los individuos bajos en $\mathrm{AO}$, por otra parte, deben mostrar menos responsividad a indicios situacionales e interpersonales relacionados con lo adecuado de su conducta, ser más consistentes transituacionalmente y presentar mayor conexión entre sus actitudes internas y su conducta (Snyder, 1979).

Una parte muy significativa de la investigación reciente en $\mathrm{AO}$ ha estudiado los diferentes «mundos sociales» de los individuos altos y bajos en esta variable. Snyder, Simpson y Gangestad (1986) plantearon las concomitancias de esta medida con las actitudes y orientaciones hacia el sexo. Específicamente, trataron de encontrar si existía una infraestructura psicológica tras dos diferentes orientaciones hacia la sexualidad: una, que llamaron restringida, marcada por la presencia de fuertes compromisos con la pareja como requisito de la relación sexual, y otra, que se denominó amplia, que admite relaciones múltiples, menos comprometidas y con personas poco conocidas. En el cuestionario de sexualidad utilizado, se confirmó factorialmente la existencia de un factor general de orientación hacia la sexualidad, independiente de un segundo factor de frecuencia en las relaciones sexuales. Coherentemente con las características de la variable de $\mathrm{AO}$, se postuló que los sujetos que puntuaban alto en esta variable, tenderían a responder afirmativamente a aquellas cuestiones que revelasen una orientación amplia, mientras que los sujetos que puntuasen bajo en $\mathrm{AO}$ se mostrarían más proclives a las relaciones sexuales restringidas. Los resultados confirmaron estas hipótesis.

Desde hace varios años hemos utilizado, con fines de investigación, la escala de AO de Snyder con universitarios. La composición de esta escala en nuestra población, aunque no ofrece una estructura homogénea, delimi- 
ta varios factores consistentes con los planteamientos de Snyder (Carrillo, Avia y Rojo, en prensa). Por otro lado, la significación psicológica de la variable $\mathrm{AO}$, si entendemos como tal su relación con otras que constituyen la personalidad, ha merecido un trabajo adicional (Avia, Carrillo y Rojo, en prensa), que no es del todo consistente con las hipótesis de Snyder.

Puesto que los resultados de Snyder y cols. sobre orientación sexual confirman la relevancia de la $\mathrm{AO}$ en el área de las relaciones interpersonales, nos planteamos aplicar su misma encuesta a una población española equivalente (estudiantes de Psicología). Tres diferencias notables existen entre el trabajo de Snyder y cols. y el que a continuación presentamos. En primer lugar, en nuestra muestra se incluyen estudiantes de todós los cursos de Psicología, ya que consideramos que las diferencias de edad deben ser predictoras de las respuestas al cuestionario de sexualidad. En segundo lugar, aunque casi todas las predicciones de Snyder se presumen «incontaminadas» por el sexo, y el peso que se concede a esta variable es muy débil, consideramos que los mundos sociales e interpersonales y la orientación sexual de hombres y mujeres son, a menudo diferentes, debido en parte a la estructuración de roles en la cultura occidental. Por ello, asumimos que la orientación sexual restringida o laxa estará, en gran parte, relacionada con el sexo. Finalmente, aunque el tamaño de la muestra de Snyder y cols. y la nuestra propia es aproximadamente igual (255 sujetos en su caso y 265 en el nuestro), en la mayor parte de sus análisis se incluye únicamente el $63 \%$ de la muestra, formada por quienes informaron haber tenido experiencia de coito. En nuestro caso, hemos trabajado inicialmente con todos los sujetos, separando posteriormente la muestra según la presencia/ausencia de relaciones coitales. Estas tres diferencias nos han llevado a realizar un análisis de datos diferente al suyo, que a continuación se expone. Las hipótesis que guían nuestro trabajo sugieren que la orientación sexual restringida, marcada por la necesidad de estrechos vínculos emocionales y de compromiso para la relación sexual, estará asociada al sexo femenino, a menor edad y a bajas puntuaciones en AO. Por el contrario, los sujetos altos en $\mathrm{AO}$, varones y de mayor de edad, tenderán a mostrar actitudes secuales más laxas. Frente a la postura de Snyder, consideramos el sexo la variable más relevante a la hora de explicar las diferencias en orientación sexual.

\section{PROCEDIMIENTO Y DISEÑO EXPERIMENTAL}

269 estudiantes universitarios, 104 varones y 165 mujeres, en su mayor parte de la Facultad de Psicología de la Universidad Complutense (un $25 \%$ de la muestra la formaron estudiantes de Medicina y Veterinaria), contestaron de forma anónima y voluntaria a la escala de AO de Snyder y al cuestionario de sexualidad diseñado por Snyder y cols. (ver anexo), que fueron presentados en sobres cerrados. El rango de edad fue de 17 a 40 años. Se utilizó un diseño $2 \times 2 \times 2$, en el que se consideraron variables independientes: sexo, edad (dicotomizado entre iguales o menores de 18 $-\mathrm{N}=121-$ y mayores o iguales a 23 años $-\mathrm{N}=148$ ) y $\mathrm{AO}$ (bajos: sujetos con puntuaciones inferiores a 10; altos: sujetos superiores a dicha puntuación). De este modo, se realizaron análisis de varianza sobre las res- 
puestas a los 20 ítems de la encuesta de sexualidad, cuyos valores se consideraron como la variable dependiente.

En la tabla 1 se presentan los valores de la prueba $\mathrm{F}$ para cada ítem de la encuesta, con su nivel de significación en las tres variables principales y en sus interacciones.

TABLA I

Valores prueba $F$ (muestra total) y niveles de significación

\begin{tabular}{llllllllllllllll}
\hline & E & & & S & & AO & & ES & & EA & & SA & & ESA \\
\hline & F & p & F & p & F & p & F & p & F & p & F & p & F & p \\
\hline
\end{tabular}

ITEMS CONDUCTUALES

$\begin{array}{rrrrrrrrrrrrrrr}1 & 23.2 & .00 & 1.7 & - & 1.4 & - & 2.1 & - & .1 & - & .3 & - & .02 & - \\ 2 & 14.3 & .00 & 6.1 & .01 & 1.2 & - & 1.2 & - & 2.1 & - & .0 & - & .59 & - \\ 3 & 7.3 & .00 & 8.8 & .00 & .3 & - & 3.1 & - & 1.3 & - & .0 & - & 1.4 & - \\ 4 & 19.1 & .00 & 3.2 & .07 & 2.4 & - & 10.3 & .00 & .0 & - & .7 & - & .00 & - \\ 5 & .9 & - & 44.3 & .00 & 4.1 & .0 & .2 & - & 1.9 & - & 1.0 & - & .3 & - \\ 6 & 3.5 & .06 & 7.2 & .00 & 8.6 & .00 & .6 & - & .0 & - & .2 & - & .00 & - \\ 7 & 3.1 & .08 & 1.7 & - & 1.1 & - & 2.0 & - & .9 & - & .4 & - & .6 & - \\ 8 & 5.3 & .02 & 1.2 & - & 1.0 & - & 3.6 & - & .7 & - & .2 & - & .8 & - \\ 9 & 2.3 & - & 15.9 & .00 & 4.6 & .03 & .4 & - & .1 & - & .2 & - & .4 & - \\ 10 & .1 & - & 22.5 & .00 & 7.3 & .00 & .1 & - & .2 & - & .7 & - & .00 & -\end{array}$

INDICES ACTITUDINALES

$\begin{array}{rrrrrrrrrrrrrrr}1 & 3.4 & .07 & 6.3 & .01 & .1 & - & .4 & - & .0 & - & .3 & - & 2.9 & .09 \\ 2 & 6.5 & .01 & 7.6 & .00 & 3.9 & .05 & 4.3 & .04 & .4 & - & .6 & - & .1 & - \\ 3 & 2.0 & - & 34.9 & .00 & 3.7 & .05 & 1.6 & - & .5 & - & .1 & - & .15 & - \\ 4 & .3 & - & 24.7 & .00 & 1.9 & - & .2 & - & 1.5 & - & .4 & - & 1.9 & - \\ 5 & 3.1 & - & 25.5 & .00 & .7 & - & .1 & - & .3 & - & .11 & - & .01 & - \\ 6 & 2.6 & - & 21.9 & .00 & 5.8 & .02 & .2 & - & .1 & - & .19 & - & .02 & - \\ 7 & 1.4 & - & 11.7 & .00 & 5.5 & .02 & 2.3 & - & 1.8 & - & .09 & - & 2.2 & - \\ 8 & 2.4 & - & 16.6 & .00 & 2.1 & - & 1.4 & - & .4 & - & .23 & - & 3.5 & .06 \\ 9 & 14.85 & .0 & 10.9 & .00 & 1.3 & - & 2.78 & .09 & .1 & - & .26 & - & .07 & - \\ 10 & 4.78 & .03 & .7 & - & 11.6 & .00 & .18 & - & 10.9 & .00 & 1.0 & - & .56 & -\end{array}$

Item control

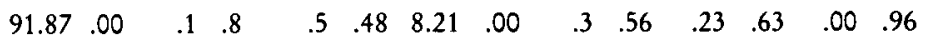

LEYENDA: $\mathbf{E}=$ Edad; $\mathrm{S}=$ Sexo; $\mathrm{AO}=$ Auto-Observación $; \mathrm{ES}=$ Interacción Edad $/$ Sexo; EA = Interacción Edad/AutoObservación; $\mathrm{SA}=$ Interacción Sexo/Auto-Observación; ESA = Interacción triple.

\section{RESULTADOS}

\section{Items conductuales:}

\section{Item 1: Frecuencia de relaciones el mes pasado}

La única variable que determina la frecuencia de las relaciones sexuales es la EDAD: a más EDAD, más relaciones sexuales $(\mathrm{F}(1,261)=23.23$, $\mathrm{p}=.000$ ). Ni el sexo ni la auto-observación, como ocurrió con los datos de Snyder alcanzaron el nivel de significación. 
Item 2: Práctica del sexo oral

La edad $(F(1,261)=14.31, p=.0002)$ y el sexo $(F(1,261)=6,15$, $p=013)$ marcan las diferencias en este item: los mayores y los varones han practicado más el sexo oral que los más jóvenes y las mujeres.

Item 3: Número de parejas diferentes a lo largo de la vida

Edad $(F(1,261)=7.29, \mathrm{p}=.007)$ y sexo $(\mathrm{F}(1,261)=8.84, \mathrm{p}=.003)$ alcanzan significación, siendo los mayores y los varones quienes han tenido relaciones múltiples en su vida. Aparece una interacción significativa $(F(1,261)=3.10, p=.07)$ entre edad y sexo.

Item 4: Número de parejas diferentes en el pasado año

Se obtienen resultados semejantes al ítem anterior (edad: $F(1,261)=19.05, p=.000 ;$ sexo: $F(1,261)=3.23, p=.07 ;$ edad $/$ sexo: $\mathrm{F}(1,261)=10.31, \mathrm{p}=.001)$.

Item 5: Número de "posibles parejas" si se eliminaran fuentes de temor (venéreas, rechazo, etc.)

Lo más llamativo es el importantísimo valor diferencial del sexo en las respuestas a este ítem $(\mathrm{F}(1,261)=44.27, \mathrm{p}=.000)$ : a los varones les gustaría entablar más relaciones diferentes que a las mujeres. También las diferencias de personalidad afectan a esta medida: los individuos altos en auto-observación tendrían más relaciones con diferentes parejas si se eliminasen sus miedos $(\mathrm{F}(1,261)=4.09, \mathrm{p}=.04)$.

Item 6: Personas diferentes con las que consideran probable relacionarse en los próximos cinco años

Las personas de más edad anticipan relaciones con más parejas distintas $(\mathrm{F}(1,261)=3.55, \mathrm{p}=.06)$ y más, también, los altos en auto-observación que los bajos $(F(1,261)=8.62, \mathrm{p}=.003)$.

Item 7: Número de contactos sexuales de una sola noche

La edad es la única variable que predice diferencias en esta pregunta: los mayores han tenido más contactos de este tipo $(F(1,261)=3.05$, $\mathrm{p}=.08)$.

Item 8: Relaciones con otras personas durante la relación de pareja

La edad se muestra significativa, con los mayores con más relaciones «extra» durante las relaciones de pareja $(\mathrm{F}(1,261)=5.33, \mathrm{p}=.02)$. Aparece también una interacción edad/sexo, con los varones de mayor edad puntuando más alto $(\mathrm{F}(1,261)=3.56, \mathrm{p}=.06)$.

Item 9: Frecuencia de pensamientos sobre sexo

La variable que más discrimina es el sexo: más pensamientos sexuales los varones que las mujeres $(\mathrm{F}(1,261)=15.93, \mathrm{p}=.0001)$. La AO también discrimina las respuestas a este ítem, con los individuos altos en $\mathrm{AO}$ mos- 
trando un mayor número de pensamientos sobre sexo $(F(1,261)=4.58$, $\mathrm{p}=.03)$.

Item 10: Frecuencia de fantasías sexuales con personas diferentes a la pareja habitual

El sexo es una variable muy discriminativa en las respuestas a esta pregunta $(F(1,261)=22.52, p=.000)$, en la que los varones informan de mayor número de fantasías sexuales con personas distintas a la pareja. La AO tiene también un peso importante $(F(1,261)=7.32, p=.007)$ con más fantasías los individuos que puntúan alto en esta variable.

\section{Items actitudinales:}

\section{Item 1: Las relaciones sexuales no implican compromiso}

Discriminan significativamente las respuestas a este ítem las variables de edad $(\mathrm{F}(1,261)=3.36, \mathrm{p}=.06)$, sexo $(\mathrm{F}(1,261)=6.28, \mathrm{p}=.01)$ y las interacción triple edad, sexo y $\mathrm{AO}(\mathrm{F}(1,261)=2.88, \mathrm{p}=.09)$. Muestran una actitud de menor compromiso en la relación sexual los mayores y los. varones. Respecto a la interacción, el grupo que responde más afirmativamente es el formado por varones mayores altos en auto-observación, que funciona como un subgrupo más «cínico" y menos comprometido:

Item 2: Incapacidad de mantener relaciones sexuales si no hay compromiSO

En la línea inversa al anterior, responden más afirmativamente los más jóvenes $(\mathrm{F}(1,261)=6.53, \mathrm{p}=.04)$, las mujeres $(\mathrm{F}(1,261)=7.58, \mathrm{p}=.006)$ $y$ los que puntúan bajo en $\mathrm{AO}(\mathrm{F}(1,261)=3.96, \mathrm{p}=.04)$. Hay una relación significativa edad/sexo $(\mathrm{F}(1,261)=4.31, \mathrm{p}=.03)$.

Item 3: Imaginarse a si mismo disfrutando sexualmente con diferentes relaciones esporádicas

En esta medida, un factor fuertemente discriminante es la variable sexo $(\mathrm{F}(1,261)=34.92, \mathrm{p}=.000)$ con una puntuación más alta en los varones. También es significativo el efecto de la auto-observación, con más respuestas afirmativas los altos en esta medida $(F(1,261)=3.69, \mathrm{p}=.05)$.

Item 4: Comodidad en relaciones sexuales con personas atractivas y poco conocidas

La única variable que discrimina las respuestas en esta pregunta es el sexo, siendo los varones quienes muestran una actitud más «abierta" $(\mathrm{F}(1,261)=24.69, \mathrm{p}=.000)$.

Item 5: Incomodidad en la relación sexual con alguien poco conocido

Los sujetos más jóvenes $(\mathrm{F}(1,262)=3.10, \mathrm{p}=.07)$ y las mujeres $(\mathrm{F}(1,261)=25.47, \mathrm{p}=.000)$ se sienten más incomodos en relaciones sexuales con parejas no "familiares". 
Item 6: Necesidad de fuertes lazos para acceder a la relación sexual

Las mujeres $(F(1,261)=21.93, p=.000)$, los bajos en $A O$ $(\mathrm{F}(1,261)=5.84, \mathrm{p}=.01)$ y los más jóvenes $(\mathrm{F}(1,261)=2.6, \mathrm{p}=.10)$ consideran más necesarios los vínculos emocionales y psíquicos para la relación sexual.

\section{Item 7: El sexo sin amor está muy bien}

Predicen las reacciones a esta pregunta el sexo $(F(1,261)=11.7$, $\mathrm{p}=.0007)$ y la $\mathrm{AO}(\mathrm{F}(1,261)=5.53, \mathrm{p}=.01)$ en la línea de las respuestas anteriores: los varones y los sujetos altos en auto-observación se adhieren más a esta afirmación.

Item 8: Lazos emocionales y psíquicos innecesarios para la relación sexuall

Significativamente son los varones los que admiten con mayor facilidad las relaciones sexuales sin que medien vínculos emocionales $(F(1,261)=16.6, p=.0001)$. Aparece también la interacción entre edad, sexo y auto-observación como predictora de las respuestas a este ítem $(\mathrm{F}(1,261)=3.5, \mathrm{p}=.06)$.

Item 9: Evaluación de la propia experiencia sexual en relación con la media de su edad

La edad $(F(1,261)=14.85, \mathrm{p}=.0001)$ y el sexo $(\mathrm{F}(1,261)=10.96$, $\mathrm{p}=.001)$ predicen las diferencias en esta medida. Los sujetos mayores $y$ los varones consideran que su experiencia sexual es superior a la de su grupo normativo, apareciendo, además, una interacción significativa entre ambas variables $(F(1,261)=2.78, \mathrm{p}=.09)$.

Item 10: Evaluación del propio atractivo físico en relación con personas de su edad

Los mayores $(F(1,261)=4.68, \mathrm{p}=.02)$ y los sujetos altos en $\mathrm{AO}$ $(\mathrm{F}(1,261)=11.58, \mathrm{p}=.0008)$ se consideran más atractivos que la media si se comparan con los más jóvenes y los bajos en $\mathrm{AO}$. La interacción edad/AO es también significativa $(F(1,261)=10.96, p=.001)$.

A la luz de estos resultados, parece evidente que nuestras tres variables independientes tienen un peso explicativo importante en relación con las variaciones en las respuestas al cuestionario de sexualidad. El sexo, es, en primer lugar, la variable más discriminativa, tanto por el número de relaciones significativas (en 15 de las 20 preguntas del cuestionario, 7 sobre datos conductuales y 8 actitudinales) como por el alto nivel de significación alcanzado en las pruebas " $\mathrm{F}$ ». En todos los casos, los resultados señalan un perfil más abierto y menos restringido en los varones en cuanto a sexualidad. Quizá sea más sencillo concluir que en sólo tres índices el sexo no ha resultado ser una variable discriminativa: los varones no indican una mayor frecuencia de relaciones sexuales que las mujeres, no han tenido más contactos sexuales de una sola ocasión, ni varían respecto a las mujeres en 
el mantenimiento de relaciones «extra» durante la relación de pareja. En todos los demás casos, que según se ha visto incluyen datos de conducta manifiesta (práctica del sexo oral, número de parejas diferentes en toda su vida y en el último año) y también cognitiva (frecuencia de interacciones si se eliminasen miedos, anticipación de posibles relaciones sexuales en los años futuros, fantasías sexuales con personas distintas y frecuencia de pensamientos sexuales) los varones difieren sustancialmente de las mujeres.

En segundo lugar, la edad, dentro de los márgenes de nuestra muestra, se perfila también como un factor relevante. Al tratarse de un período evolutivo central en el desarrollo de la sexualidad, las diferencias entre los más jóvenes y los mayores son también muy notables: en 7 medidas conductuales y en 5 actitudinales, sobre las 20 del total. Los datos más destacados son los siguientes: la edad es la única variable que predice la frecuencia de las relaciones sexuales en nuestra muestra, así como la existencia de relaciones de una sola noche. El conjunto de los datos actitudinales y conductuales señalan una evolución de la sexualidad hacia una mayor desimplicación en las relaciones, perdiéndose la necesidad de poseer fuertes vínculos afectivos a medida que la edad progresa. Los más jóvenes se dibujan como más idealistas y se muestran más incómodos ante posibles encuentros sexuales con personas poco conocidas. Curiosamente, a mayor edad se incrementa la sensación de experiencia sexual respecto al propio grupo de referencia, así como la autoestima sobre el atractivo físico.

Finalmente, las diferencias en personalidad discriminan las respuestas al cuestionario en 9 de los 20 ítems, siempre en la dirección apuntada en las hipótesis que guiaron nuestro trabajo,y en concordancia con los datos obtenidos por Snyder. Es interesante observar que cuatro de estos ítems afectan a medidas conductuales de tipo cognitivo (frecuencia de relaciones sexuales con personas distintas si desaparecieran los miedos, previsión de relaciones en los años próximos, frecuencia de pensamiento sobre el sexo y fantasías con personas diferentes a la pareja), otros cuadros se refieren a medidas actitudinales que reflejan una sexualidad más libre y menos comprometida en los sujetos auto-observadores, y un último ítem señala que los sujetos altos en $\mathrm{AO}$ se consideran significativamente más atractivos que los bajos. Todo parece señalar, pues, que las diferencias entre los sujetos altos y bajos en $\mathrm{AO}$ en cuanto a sexualidad son más bien diferencias en fantasía, interés por la sexualidad y actitudes ante la misma, sin que aparezcan, como tampoco ocurriera en el trabajo de Snyder, diferencias en la frecuencia de las relaciones.

\section{Subgrupos con/sin experiencia de coito}

Con el fin de poder controlar el efecto de la fantasía sin base real, se incluyó en la encuesta, como control, la pregunta de si el individuo había hecho alguna vez el amor. Unicamente la edad $(F(1,261)=91.87, \mathrm{p}=.0000)$ y la interacción edad/sexo $(F(1,261)=8.21, \mathrm{p}=.004)$ fueron significativas en esta variable. Dicotomizando por esta variable se formaron dos grupos sobre los que volvimos a analizar los resultados. El $54 \%$ de la muestra $(\mathrm{N}=146)$ informó haber tenido experiencia de coito, y el $45 \%(\mathrm{~N}=123)$ no la tuvo. Esta experiencia se dio en el $67 \%$ de los varones y en el $46 \%$ de las mujeres. 
En las tablas 2 y 3 se representan mediante asteriscos los valores «F» significativos de las tres variables independientes y sus interacciones para ambos subgrupos.

TABLA II

Items conductuales

\begin{tabular}{|c|c|c|c|c|c|c|c|c|c|c|c|c|c|c|}
\hline & \multicolumn{7}{|c|}{ Con experiencia de coito } & \multicolumn{7}{|c|}{ Sin experiencia de coito } \\
\hline & $\mathrm{E}$ & $S$ & $\mathrm{AO}$ & ES & EA & SA & ESA & $\mathrm{E}$ & S & $\mathrm{AO}$ & ES & $\mathrm{EA}$ & SA & ESA \\
\hline 1. Frecuencia rel. último mes & & & & & & & & & $*$ & & & & 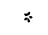 & \\
\hline 2. Sexo oral & & & & & & & & & $\nLeftarrow$ & & & $*$ & & 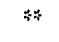 \\
\hline 3. Parejas dif. en la vida & & & & & & & & & $*$ & & & & & \\
\hline 4. Parejas dif. último año & & & & & & & & & & $"$ & & & & \\
\hline $\begin{array}{l}\text { 3. Paedos } \\
\text { mier. S1 no tuotese }\end{array}$ & & & & & & & & & $x \div$ & $\because$ & & & $*$ & \\
\hline $\begin{array}{l}\text { 6. Parejas anticipadas próxi- } \\
\text { mos } 5 \text { años } \\
\text { 7. Rel. sexuales un sólo contac- }\end{array}$ & & & & & & & & & & $\Rightarrow$ & $\because$ & & & \\
\hline to & & & & & & & & & & & $\Rightarrow$ & & & \\
\hline 8. Rel. extra pareja & & & & & & & & & & & & & & \\
\hline $\begin{array}{l}\text { 9. Frecuencia de pensamientos } \\
\text { sexuales }\end{array}$ & & & & & & & & & \&: & & & & & $*$ \\
\hline 10. Fantasías extra pareja & & 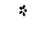 & & & & & * & & +4 & & & & & \\
\hline
\end{tabular}

Valores F significativos de las $3 \mathrm{VI}$ (edad, sexo y Auto-Observación) y sus interacciones en los ítems CONDUCTUALES de la encuesta para sujetos con/sin experiencia de coito.

* Signif. con $\mathrm{p}<.5$

$\approx$ Signif. $\operatorname{con} p<.01$

$\Leftrightarrow *$ Signif. con $p<.001$

\section{TABLA III}

Items actitudinales

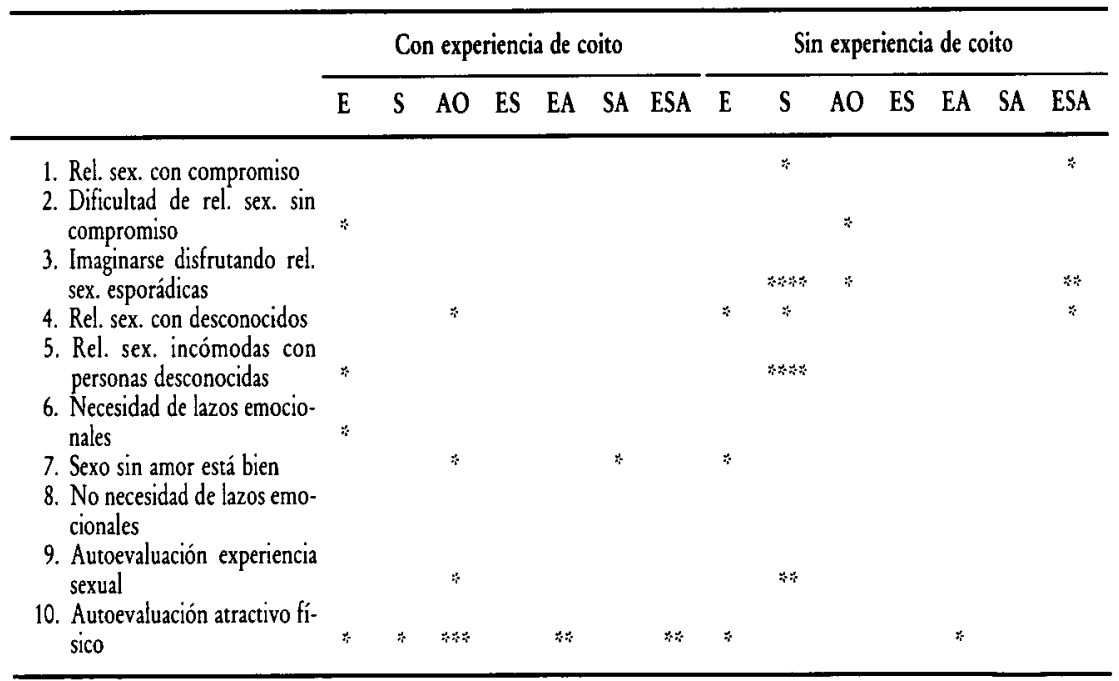

Valores F significativos de las 3 VI (edad, sexo y Auto-Observación) y sus interacciones en los ítems ACTITUDINALES de la encuesta para sujetos con/sin experiencia de coito.

" Signif. con $\mathrm{p}<.5$

$\Rightarrow$ Signif. con $p<.01$

$\Rightarrow \Rightarrow$ Signif. con $p<.001$ 


\section{Datos conductuales}

Frente a los resultados comentados en el grupo total, destaca la escasez de diferencias significativas en ninguna de las variables independientes. Tomando en conjunto las tres variables independientes sólo aparecen dos razones « $F$ » significativas en total de los 10 ítems conductuales, refiriéndose una a la variable de personalidad (los más altos en $\mathrm{AO}$ refieren tener más fantasías sexuales con parejas diferentes a la propia -item 10-: $(\mathrm{F}(1,138)=4.78, \mathrm{p}=.03)$ y otra, en este mismo ítem, a la interacción edad/sexo/auto-observación $(F(1,138)=3.08, \mathrm{p}=.08)$. En el ítem 9 (frecuencia de pensamientos sexuales) los sujetos más altos en $\mathrm{AO}$ alcanzan puntuaciones superiores, rozándose el nivel de significación $(\mathrm{F}(1,138)=2.71, \mathrm{p}=.10)$; y el ítem 5 (frecuencia de relaciones si se eliminasen los miedos) el sexo se aproxima, asimismo, al nivel de significación $(\mathrm{F}(1,138)=2.7, \mathrm{p}=.10)$. Por tanto, el tremendo poder diferencial del sexo en el grupo total desaparece absolutamente al considerar únicamente los sujetos con experiencia coital, y lo mismo cabe decir de la variable edad. Sólo la $\mathrm{AO}$ y en mucha menor medida que entonces, conserva un cierto, aunque débil, poder diferenciador.

\section{Datos actitudinales}

Los resultados son semejantes a los comentados anteriormente aunque aparecen algunas relaciones más. La edad ofrece un valor diferenciador en 5 ítems $(2,4,5,6$ y 10) siendo los más jóvenes los más incómodos en relaciones sexuales $\sin$ compromiso $(\mathrm{F}(1,138)=3.82, \mathrm{p}=.05)$ y con personas poco conocidas $(\mathrm{F}(1,138)=3.99, \mathrm{p}=.04)$, mostrando los mayores más comodidad en éstas últimas $(F(1,138)=3.17, p=.07)$ y necesitando menos lazos emocionales para la relación sexual $(\mathrm{F}(1,138)=3.45, \mathrm{p}=.06)$. La variable $\mathrm{AO}$ ofrece, asimismo, cinco relaciones diferenciales en los ítems 4 , $6,7,9$ y 10: los más auto-observadores se sienten mejor con personas atractivas poco conocidas $(\mathrm{F}(1,138)=3.58, \mathrm{p}=.06)$, necesitan menos lazos afectivos para el sexo $(\mathrm{F}(1,138)=3.15, \mathrm{p}=.07)$, afirman en mayor frecuencia que el sexo sin amor está muy bien $(\mathrm{F}(1,138)=5.05, \mathrm{p}=.02)$ y evalúan mejor su propia experiencia sexual en relación al grupo $(F(1,138)=3.8$, $\mathrm{p}=.05$ ). Finalmente, sólo en el ítem 10 (evaluación del propio atractivo físico) arroja el sexo una relación significativa en el sentido de evaluarse más positivamente las mujeres que los hombres con experiencia sexual. Curiosamente, en esta pregunta muestran razones « $\mathrm{F} »$ significativas todas las variables (edad: $F(1,138)=3.6, p=.05 ;$ sexo: $F(1,138)=3.55, p=.06 ; \mathrm{AO}$ : $\mathrm{F}(1,138)=9.12, \mathrm{p}=.003$ ) así como las interacciones (edad/sexo: $\mathrm{F}(1,138)=4.75, \mathrm{p}=.03 ; \mathrm{edad} / \mathrm{AO}: \mathrm{F}(1,138)=6.06, \mathrm{p}=.01$; $\mathrm{edad} / \mathrm{sexo} / \mathrm{AO}: \mathrm{F}(1,138)=6.7, \mathrm{p}=.01$.

Si tomamos en conjunto los datos actitudinales y los conductuales, se puede concluir que la variable sexo en la muestra de sujetos con experiencia coital, y frente a las 15 relaciones significativas de la muestra total, sólo ofrece una relación significativa, con un nivel de significación débil, y en un ítem bastante marginal, la evaluación del atractivo físico. La edad presenta, en conjunto, cinco relaciones significativas (frente a doce el análisis 
anterior), todas en índices actitudinales y con bajos niveles de significación, siendo las diferencias en auto-observación las que conducen a más polaridad de respuestas en el grupo con experiencia de coito (siete diferencias en total, dos de ellas en conducta y cinco en actitudes - dos menos que en la muestra completa-).

\section{Subgrupo sin experiencia coital}

\section{Datos conductuales}

En este grupo de sujetos vuelve a ser muy significativo el impacto de la variable sexo que, al igual que en la muestra total, discrimina en 7 de las 8 preguntas sobre datos de conducta sexual. Aunque los resultados van en la misma dirección que en el grupo conjunto, el hecho de no haber tenido este grupo experiencia de coito, hace pensar que se dan en este caso unas ciertas tendencias hacia la fantasía y por tanto, una cierta distorsión. Los varones sin experiencia de coito señalan haber tenido más experiencia sexual que las mujeres $(\mathrm{F}(1,115)=5.66, \mathrm{p}=.01)$, haber practicado más el sexo oral $(\mathrm{F}(1,115)=6.19, \mathrm{p}=.01)$, haber tenido más parejas a lo largo de su vida $(\mathrm{F}(1,115)=5.04, \mathrm{p}=.02)$, les apetecería tener más relaciones sexuales si no tuviesen los miedos habituales $(\mathrm{F}(1,115)=15.71, \mathrm{p}=.0001)$, dicen haber tenido más relaciones de una sola noche $(F(1,115)=2.86, p=.09)$, tienen con mayor frecuencia pensamientos sobre sexo $(F(1,115)=11.14$, $\mathrm{p}=.001)$ y más fantasías con personas distintas de la pareja $(\mathrm{F}(1,115)=12.47, \mathrm{p}=.0006)$.

Por el contrario es llamativo que en este grupo de personas, la edad no predice ninguna de las respuestas conductuales al cuestionario, figurando sólo como significativas tres interacciones con las otras dos variables - edad y $\mathrm{AO}$ - Esto parece contradecir el supuesto, de sentido común, de que la no experiencia coital tenga distintas repercusiones en personas de más edad que en las más jóvenes. Finalmente, la variable $\mathrm{AO}$ mantiene cuatro relaciones significativas, como ocurrió en la muestra completa, asimismo, en la línea de una mayor fantasía sexual para los sujetos altos en $\mathrm{AO}$, que dicen haber tenido más parejas diferentes en el último año $(F(1,115)=3.73$, $\mathrm{p}=.05)$ tendrían más parejas en los próximos $(\mathrm{F}(1,115)=8.6, \mathrm{p}=.004) \mathrm{y}$ mencionan mayor frecuencia de pensamientos sobre el sexo $(\mathrm{F}(1,115)=4.07, \mathrm{p}=.04)$.

Es destacable también el hecho de ser este grupo en el que aparecen más interacciones entre las variables: ocho en total, seis dobles $(3 \mathrm{sexo} / \mathrm{AO}, 2$ edad/sexo y edad/AO) y dos triples. El sentido de estas interacciones es complejo, pero apunta en la línea de la «fantasía» a la que nos referíamos más arriba. Por ejemplo, en el ítem 6, previsión de parejas en los próximos años, fantasean más relaciones los varones más jóvenes y las mujeres más mayores; en el ítem 9 (frecuencia de pensamientos sexuales) puntúan más alto lós varones más jóvenes altos en $\mathrm{AO}$, y lo mismo ocurre con el ítem 2.

\section{Datos actitudinales}

La diferenciación que ofrecía la variable sexo en los datos actitudinales en la muestra total se mantiene en este grupo: 8 de los 10 ítems actitudinales diferencian hombres y mujeres y son las mismas que se comentaron 
entonces: los varones aceptan más las relaciones sexuales sin compromiso $(\mathrm{F}(1,115=4.6, \mathrm{p}=.03)$, mostrándose las mujeres más incómodas si no lo hay $(\mathrm{F}(1,115)=2.81, \mathrm{p}=.09)$, ellos se muestran más dispuestos a relaciones con personas atractivas y poco conocidas $(F(1,115)=5.04, \mathrm{p}=.02)$ y las mujeres sienten mayor incomodidad en esas relaciones $(F(1,115)=3.47$, $\mathrm{p}=.06)$. Ellas necesitan más lazos afectivos para la relación sexual $(F(1,115)=14.21, p=.0003)$, pueden imaginarse con mayor dificultad disfrutando relaciones sexuales esporádicas $(\mathrm{F}(1,115)=22.37, \mathrm{p}=.0000)$ y se adhieren menos a la expresión «el sexo sin amor está muy bien» $(\mathrm{F}(1,115)=4.67, \mathrm{p}=.03)$. Es interesante la respuesta al ítem 9, que evalúa la propia experiencia sexual en relación al grupo: los varones sin experiencia de coito consideran que su experiencia es superior a la del grupo en mayor medida que las mujeres $(F(1,115)=6.35, p=.01)$. Esto coincide con la tendencia ya comentada a fantasear, y que parece superior en los varones que en las mujeres.

La tendencia a la fantasía y sesgo de los datos se constata al encontrar el peso de la variable edad en este mismo ítem: los más jóvenes piensan que tienen más experiencia que los mayores $(\mathrm{F}(1,115)=2.76, \mathrm{p}=.09)$. Esta variable también es significativa cuando se evalúa la incomodidad en relaciones con personas desconocidas, mostrándose los más jóvenes menos cómodos en estas relaciones $(F(1,115)=5.04, p=.02)$. En cambio, afirman más acuerdo con la idea del sexo sin amor $(F(1,115)=4.67, p=.03)$, lo cual hace sospechar que la muestra de jóvenes no experimentados, aunque responde de modo más entusiasta a "declaraciones de principios», luego se sienten más incómodos practicando esas relaciones. Finalmente, la variable AO mantiene tres relaciones significativas, que presentan de nuevo al sujeto con puntuaciones altas con una actitud más libre y menos comprometida personalmente con el sexo (ver en la tabla los ítems 2,3 y 6). Aparecen en estos datos tres interacciones triples en la línea de las predicciones teóricas.

\section{DISCUSION}

Los resultados encontrados al analizar la totalidad de la muestra han revelado que la orientación y actitud hacia la sexualidad están, sobre todo, determinados por el sexo. De modo coherente con el estereotipo social, parece que los varones tuvieran más claros, formados y accesibles esquemas sobre sexualidad y pensamientos sexuales más frecuentes, lo cual les predispondría a percibir posibilidades de encuentro en más ocasiones, y a estar más dispuestos para el sexo. Puesto que son, además, menos selectivos y pueden imaginarse disfrutando de relaciones esporádicas con personas diferentes, están más «listos para la acción», lo que parece confirmado por el mayor número de varones que de mujeres con experiencia de coito, en comparación con el $\mathrm{N}$ respectivo.

La importancia de la variable sexo, que predecía las respuestas a 15 de las 20 preguntas del cuestionario, queda, sin embargo, reducida a una diferencia marginal en la percepción del propio atractivo cuando nos centramos en el grupo con experiencia de coito. Las mujeres con esta experiencia se asemejan en todo a sus compañeros, sin que puedan distinguirse de ellos 
en fantasía sexual, conducta o actitudes. Una posible explicación de estos datos (consistentes con los resultados de Snyder y cols., puesto que sólo incluyen en sus análisis los datos de este subgrupo), vendría a señalar que aunque las actitudes hacia la sexualidad están muy marcadas en los sexos, las fantasías sexuales iniciales se modifican tras las relaciones sexuales con coito, que actuarían como "pruebas de realidad». Estas pruebas de realidad marcarían una evolución en las mujeres hacia una mayor sexualización, haciéndoles más proclives a una sexualidad menos restringida, más libre de compromisos afectivos y psicológicos, y en cierto modo, más "masculina" y cínica. Las diferencias marcadas entre los hombres y las mujeres sin experiencia de coito se reducirían, por tanto, en cuanto éstas adquiriesen experiencia. Esto coincide con las sugerencias de que la práctica sexual es importante para mantener actitudes menos restringidas ante el sexo. En el subgrupo sin experiencia de coito, las diferencias entre varones y mujeres, tanto en actitudes como en conducta, son tan fuertes como en el grupo total.

Esta explicación, que concede gran importancia a la experiencia, compite con una segunda, que no puede excluirse por completo, y es la de que los grupos con/sin experiencia se diferencian entre sí por otras características fundamentales, además de la práctica. Diferencias de personalidad, habilidades sociales, tendencias neuróticas, defectos físicos, o atractivo sexual, pueden estar asociados a una mayor o menor experiencia en las relaciones sexuales. De éstas, solamente poseemos información sobre el atractivo físico autopercibido por ambos subgrupos. Las diferencias de medias en el atractivo sexual entre los grupos con/sin experiencia sexual de coito son significativas, tanto para varones como para mujeres, y en dirección opuesta: mientras las mujeres con experiencia se ven más atractivas que las que no la tienen, son los hombres no experimentados quienes se consideran más atractivos que sus colegas con experiencia (varones: $\mathrm{RC}=3.8, \mathrm{p} .01$ ); mujeres: $\mathrm{RC}=2.05, \mathrm{p}=.05$ ). Es imposible saber si las mujeres con experiencia son más atractivas, y de ahí su éxito, o si la propia relación sexual las hace sentirse más atractivas. Los resultados en varones quizá indiquen una cierta distorsión en los no experimentados, pues parece poco plausible que la relación sexual signifique, en su caso, una pérdida de atractivo. En todo caso, parece necesario concluir que cualquier trabajo que trate de analizar diferencias en conducta y actitudes sexuales debe incluir la variable sexo. La anulación de las diferencias en los grupos más experimentados sugiere interesantes reflexiones sobre los estereotipos masculino y femenino relativos a la sexualidad.

Aunque en menor medida que en el caso de la variable sexo, la orientación sexual laxa o restringida está a su vez explicada por la edad, siendo los sujetos más jóvenes más idealistas y los mayores los que necesitan menor compromiso para disfrutar de la sexualidad. Los más jóvenes e inexpertos muestran una cierta distorsión en sus respuestas: mientras que, en general, la evaluación de su propia experiencia tiende a crecer con la edad, estos sujetos se consideran más experimentados que los mayores, dicen sentirse bien en relaciones sexuales con personas poco conocidas y se adhieren a la teoría del sexo sin amor. Todo parece indicar que este grupo suple su inexperiencia mostrando dogmáticamente actitudes contradictorias con los datos que ellos mismos ofrecen y con los de sus compañeros más experi- 
mentados, quienes reflejan fielmente la idealización del sexo en los jóvenes y las actitudes más frías y menos implicadas de los más mayores.

Consistente con los datos de Snyder, las diferencias en AO se relacionan con actitudes y conductas sexuales distintas, en la línea de la dimensión abierta/restringida que subyacía a la encuesta. Aunque en todos los casos estas relaciones van en la dirección propuesta, nuestros resultados muestran que están mediatizados por la experiencia sexual, lo cual contradice la reciente interpretación biológica de la Auto-Observación (Snyder y Gangestad, 1986; Snyder, Simpson y Gangestad, 1986). Estos datos revelan que, mientras las diferencias en actitudes y conducta sexual en el subgrupo sin experiencia de coito se explican sobre todo por el sexo, las variaciones en el subgrupo experimentado deben atribuirse fundamentalmente a diferencias en personalidad (ver tablas 2 y 3). Dado que pocas variables de personalidad se han relacionado con la sexualidad, a excepción de los trabajos de Eysenck que la conecta débilmente con extroversión, este resultado constituye un apoyo a los trabajos de Snyder y cols., que ven en los dos polos de la dimensión $\mathrm{AO}$ dos estilos interpersonales distintos. La escala de Snyder, que en este caso ha mostrado un importante valor diferencial relativo a dos distintas maneras de vivir la sexualidad puede ser utilizada para estudiar, también, otras relaciones importantes en la vida íntima, tales como la amistad, el compañerismo y el amor romántico.

\section{Referencias}

Avia, M. D., Carrillo, J. M. y Rojo, N. (en prensa). La Auto-Observación en la estructura de la personalidad.

Carrillo, J. M., Avia, M. D. y Rojo, N. (en prensa). La medida de la Auto-Observación.

GofFman, E. (1959). The Presentation of Self in everyday life. Garden City. Nueva York, Doubleday (Anchor books).

BRISSETT, D. y EDGLEY, C. (1975). Life as a theatre: a dramaturgical source book. Chicago, Aldine.

SNYDER, M. (1987). Public Appearances, Private Realities. The Psychology of Self-Monitoring. Freeman and Company, Nueva York.

- (1974). The Self-Monitoring of expressive behavior. Journal of Personality and Social Psychology, 30, 526-537.

- (1979). Self Monitoring processes. En Berkowitz, L. (Ed.). Advances in Experimental Social Psychology, vol. 12, Nueva York, Academic.

SNYDER, M. y GANGESTAD, S. (1986). On the nature of Self Monitoring; matters of assessment, matters of validity. Journal of Personality and Social Psychology, 51, 125-139.

SNYDER, M., Simpson, J. A. y GANGestad, S. (1986). Personality and sexual relations. Journal of Personality and Social Psychology, 51, 181-190. 


\section{Cuestionario sobre sexualidad}

\section{ITEMS CONDUCTUALES}

1. ¿Cuántas veces has tenido relaciones sexuales el mes pasado?

2. ¿Has practicado alguna vez el sexo oral?

3. ¿Con cuántas parejas diferentes has tenido relaciones sexuales a lo largo de tu vida?

4. ¿Con cuántas parejas diferentes has tenido relaciones sexuales el pasado año?

5. Si no tuvieras miedo a contraer enfermedades venéreas, al embarazo o a ser rechazado/a, ¿con cuántas personas que conoces te apeteceria hacer el amor?

6. ¿Con cuántas personas distintas prevees que vas a tener algunas relación sexual en los próximos cinco años?

7. ¿Con cuántas personas diferentes has tenido un sólo contacto sexual, en una sola ocasión?

8. ¿Has tenido relaciones sexuales con otrals personas mientras mantenías una relación de pareja?

9. ¿Con qué frecuencia piensas en el sexo?

Casi nunca Casi todo el tiempo

$$
\begin{array}{lllllllll}
1 & 2 & 3 & 4 & 5 & 6 & 7 & 8 & 9
\end{array}
$$

10. ¿Con qué frecuencia tienes fantasías sexuales con otra u otras personas distintas de tu pareja habitual?

Nunca Al menos una vez al día

$$
\begin{array}{llllllll}
1 & 2 & 3 & 4 & 5 & 6 & 7 & 8
\end{array}
$$

\section{INDICES ACTITUDINALES}

(Los sujeros contestan en un rango de 0 a 9 donde el cero representa no estar de acuerdo en absoluto y nueve estar totalmente de acuerdo)

1. Para mí, tener relaciones sexuales con alguien no implica necesariamente que esté comprometido/a con esa persona.

2. Yo no podría tener relaciones sexuales con alguien, a menos que antes estuviese completa y exclusivamente comprometido/a con esa persona.

3. Puedo imaginarme a mí mismo/a disfrutando de una relación sexual esporádica, con diferentes personas.

4. Podría sentirme bien teniendo una relación sexual con una persona que me resultase atractiva aunque no la conociese bien.

5. Aunque me sintiera físicamente atraído/a por una persona, me sentiría incómodo/a teniendo una relación sexual sin conocer muy bien a esa persona.

6. Tendría que estar fuertemente unido/a a una persona (emocional y psicológicamente) antes de que pudiera sentirme cómodo/a disfrutando de una relación sexual con él o ella. 
7. El sexo sin amor está muy bien.

8. Yo no tendría que estar necesariamente unido/a (emocional y psicológicamente) a una persona para iniciar con él o ella una actividad sexual.

9. ¿Cómo evalúas tu experiencia sexual?

Tengo mucha menos experiencia Tengo mucha más experiencia que la que la mayoría de la gente de mi mayoría de la gente de mi edad. edad.

$$
\begin{array}{llllll}
0 & 1 & 2 & 3 & 4 & 5
\end{array}
$$

10. ¿Cómo evalúas tu propio atractivo físico comparándote con otra gente de tu edad?

Nada atractivo

Muy atractivo

$$
\begin{array}{lllllll}
1 & 2 & 3 & 4 & 5 & 6 & 7
\end{array}
$$

- ¿Has hecho el amor alguna vez? 